\title{
Preparation and characterization of dopamine-sodium carboxymethyl cellulose hydrogel
}

\author{
Yining Chen ${ }^{1,2}$. Guolian Cui ${ }^{1,2} \cdot$ Nianhua Dan ${ }^{1,2} \cdot$ Yanping Huang ${ }^{1,2} \cdot$ Zhongxiang Bai $^{1,2} \cdot$ Changkai Yang $^{1,2}$. \\ Weihua Dan ${ }^{1,2}$
}

(c) Springer Nature Switzerland AG 2019

\begin{abstract}
In order to prepare a biocompatible tissue adhesive and sealant with high adhesion properties, dopamine (DA) was grafted onto sodium carboxymethyl cellulose (CMC) to obtain catechol-modified CMC-DA by carbodiimide chemistry method, and then CMC-DA hydrogels was prepared by self-cross-linking. The UV-Vis spectroscopy, FTIR and ${ }^{1} \mathrm{H}$ NMR results showed that dopamine was successfully introduced into CMC and the degree of substitution of dopamine in the CMC-DA hydrogels was $5 \%, 10.5 \%$ and $15 \%$, respectively. SEM observation indicated that CMC-DA possessed porous structures. Cytotoxicity experiments showed that CMC-DA has good biocompatibility. The introduction of DA could further improve the biocompatibility of hydrogel. Bulk adhesion property of the hydrogels was studied by lap shear tests. Results showed that the adhesion strength of CMC was improved indeed after modified by dopamine.
\end{abstract}

Keywords Dopamine $\cdot$ Sodium carboxymethyl cellulose $\cdot$ Hydrogel $\cdot$ Bio-adhesion

\section{Introduction}

Sutures are commonly used for wound closure and bleeding control; however they still have limitations such as, high infection rate, inconvenience in handling, and concern over possible transmission of blood-borne disease through the use of needles. One of the challenges of tissue engineering consist on the design and development of biocompatible tissue adhesives and sealants with high adhesion properties to repair or attach devices to tissues $[1,2]$. Biological adhesive is a kind of biomedical material that can be filled, closed and glued to the wound. It has both the bonding properties of general adhesives and other functions to meet the requirements in biomedical applications [3, 4].

Sodium carboxymethyl cellulose (CMC) is a derivative of cellulose after carboxymethylation, also known as cellulose gum, a kind of natural polysaccharide, which has been widely used in the fields of food, medicine, paper, chemical, and so on [5-7]. Because of its good biocompatibility, low toxicity, low immunogenicity and biodegradability, CMC has been widely applied in the field of tissue engineering $[8,9]$. CMC belongs to the anionic linear polymer material, which has good water solubility during to the carboxymethyl structure on the piranosine unit and can form a transparent viscous liquid after dissolving in water. In view of the low adhesion strength of single CMC and its large number of active groups such as carboxyl and hydroxyl groups, it is necessary to improve adhesion properties and biocompatibility through modification.

Dopamine (3,4-dihydroxyphenylethylamine, DA) is a derivative of tyrosine in mussel mucin containing catechol functional groups, giving it unique bioadhesive properties $[10,11]$. In recent years, dopamine has been widely used for the preparation of bio-adhesives primarily inspired by the superior adhesion of mussels in the ocean [12]. Mussel

\footnotetext{
$\bowtie$ Nianhua Dan, dannianhua@scu.edu.cn; $\bowtie$ Weihua Dan, danweihua_scu@126.com | ${ }^{1}$ Key Laboratory for Leather Chemistry and Engineering of the Education Ministry, Sichuan University, Chengdu 610065, Sichuan, China. ${ }^{2}$ Research Center of Biomedical Engineering, Sichuan University, Chengdu 610065, Sichuan, China.
} 
is a kind of marine organism widely existing on the coast and offshore. It can rely on the "foot plate" formed by secreted filaments to adhere firmly to various inorganic and organic surfaces in seawater [13]. The strong adhesive properties possessed in wet conditions are in line with the requirements for the use of tissue adhesives. Bio-adhesives based on biomimetic studies of mussel foot pad are considered to be the most suitable tissue adhesives for clinical needs [14].

The combination of dopamine and CMC can improve the adhesive properties and biocompatibility of natural polysaccharides. In this study, the amino groups of dopamine and the carboxyl groups of CMC were cross-linked by an amidation reaction under the action of carbodiimide (EDC). Thus, dopamine was introduced into the CMC molecular chain to prepare a new type of bio-adhesive hydrogel after self-cross-linking, and the physicochemical properties, adhesion strength and biocompatibility of the bonded hydrogel were characterized.

\section{Experimental}

\subsection{Materials}

Dopamine hydrochloride and carboxymethyl cellulose sodium (65-80\% carboxyl degree, average $M_{w}$ was about 250,000 , Sodium (ICP) was $6.5-9.5 \%$ ) were purchased from Sigma-Aldrich (St. Louis, MO, USA); 1-(3-dimethylaminopropyl)-3-ethylcarbodiimide (EDC), $\mathrm{N}$-hydroxysuccinimide (NHS) and 2-( $\mathrm{N}$-morpholine) Ethanesulfonic acid (MES) were purchased from Gongjia Chemical Technology Co., Ltd. (Shanghai, China), gelatin was purchased from Jinshan Chemical Reagent Co., Ltd. (Chengdu, China). Unless otherwise indicated, other chemicals and reagents were purchased from Kelong Chemical Reagent Co., Ltd. (Chengdu, China). Mouse fibroblast L929 was purchased from Huaxi State Key Laboratory of Biotherapy (Chengdu, China).

\subsection{Preparation of hydrogels with different degrees of dopamine substitution}

The CMC was weighed and put in a three-necked flask with $100 \mathrm{ml}$ of MES buffer solution. The mixture was stirred under a $35^{\circ} \mathrm{C}$ water bath, resulting in complete dissolution of the $\mathrm{CMC}$ to a mass fraction of 2.5\%. A certain amount of NHS and EDC was added in order to activate the cross-linking reaction, after $20 \mathrm{~min}$, dopamine was added. The $\mathrm{pH}$ of the reaction system was adjusted to about 5.0 with dilute hydrochloric acid. Nitrogen protection was always applied throughout the reaction and the reaction time lasted $24 \mathrm{~h}$. After the reaction was completed, the reaction product was loaded into a dialysis bag with a molecular weight of $3000 \mathrm{Da}$, dialyzed against distilled water for 3 days, and changed every 4-5 times. The sample was then freeze-dried and stored for future use. Before use, the lyophilized CMC-DA sample was dissolved in a phosphate solution (PB) to make a $20 \%(\mathrm{w} / \mathrm{w})$ solution, and the solution was stored at room temperature for a period of time to obtain the adhesive hydrogel which was labeled as CMC-DA.

\subsection{UV-visible spectroscopic analysis}

Freeze-dried CMC-DA was dissolved in PB buffer to prepare a solution with a mass concentration of $0.25 \mathrm{mg} / \mathrm{mL}$. A full-spectrum scan was performed in an ultraviolet-visible spectrophotometer (LAMBDA25, PE CO., American) and the absorbance values at $280 \mathrm{~nm}$ were measured. The degree of substitution of dopamine in CMC-DA was determined using a standard curve method.

\subsection{Measurement of FTIR spectra}

The approximately $2 \mathrm{mg}$ of each specimen mixed with about $100 \mathrm{mg} \mathrm{KBr}$ were pressed into tablets. Fourier transform infrared (FTIR) spectra were measured from the tablets using a FTIR spectrophotometer (Nicolet iS10, Thermo Scientific CO., America). The spectra were collected from 4000 to $400 \mathrm{~cm}^{-1}$ at a rate of 32 scans per spectrum and a resolution of $4 \mathrm{~cm}^{-1}$ at room temperature with a humidity of around $65 \%$.

\section{$2.5{ }^{1} \mathrm{H}$ NMR spectroscopy}

CMC-DA samples were dissolved in $\mathrm{D}_{2} \mathrm{O}$ respectively, and the ${ }^{1} \mathrm{H}$ NMR spectra were carried out on a Bruker AVII$400 \mathrm{MHz}$ (Bruker, Switzerland).

\subsection{Determination of equilibrium swelling ratio}

The lyophilized CMC-DA hydrogel sample was weighed and recorded as $m_{d}$, then immersed in a PB buffer solution and placed in a $37^{\circ} \mathrm{C}$ water bath to swell the hydrogel to equilibrium. After adsorption water on the surface of the sample was removed with filter paper, sample was weighed and recorded as $m_{w}$. Three parallel data were measured for each hydrogel sample. The equilibrium swelling ratio (SR) was calculated according to the following formula [15]:

$\operatorname{SR}(\%)=\frac{\left(m_{w}\right)-\left(m_{d}\right)}{m_{d}} \times 100$ 


\subsection{Micromorphology analysis}

The morphologies of CMC-DA hydrogel samples were characterized by scanning electron microscopy (JSM7500F, JEOL, Japan). The specimens were sputter-coated with aurum, and the images were acquired at an accelerating voltage of $5 \mathrm{kV}$.

\subsection{Evaluation of in vitro biocompatibility}

The biocompatibilities of CMC-DA hydrogel samples were investigated with L929 fibroblast cells using cell proliferation assay and confocal laser scanning microscopy (CLSM). Briefly, the samples were sealed and sterilized using a ${ }^{60} \mathrm{Co}$ irradiation, and thereafter were soaked in culture media and incubated at $37^{\circ} \mathrm{C}$ for $24 \mathrm{~h}$, to prepare sample solutions. In the meantime, L929 fibroblast cells were cultured in RPMI 1640 medium supplemented with $1 \%(\mathrm{v} / \mathrm{v})$ antibiotic solution and $10 \%$ calf serum in 96 -well plates, for $24 \mathrm{~h}$ at $37{ }^{\circ} \mathrm{C}$ in $5 \% \mathrm{CO}_{2}$ atmosphere. After that, the culture medium was replaced with the sample solution, and then further incubated for additional 1, 3, and 5 days at $37^{\circ} \mathrm{C}$ in $5 \% \mathrm{CO}_{2}$ atmosphere. At each time point, $20 \mu \mathrm{l}$ of 3-(4,5-dimethylthiazol-2yl)-2,5-diphenyltetrazoliumbromide (MTT) was added, and incubated at $37{ }^{\circ} \mathrm{C}$ for $4 \mathrm{~h}$ to allow the formation of formazan crystals. Subsequently, DMSO (200 $\mu \mathrm{l} /$ well) was added to dissolve the formazan crystals, and the optical density (OD) at $492 \mathrm{~nm}$ was then measured using a microplate reader (Model 550, Bio Rad Corp. USA). The biocompatibility of CMC-DA hydrogel samples was further evaluated and observed by CLSM. Briefly, L929 fibroblasts were cultured on pADM scaffolds for 3 days at $37^{\circ} \mathrm{C}$ in $5 \% \mathrm{CO}_{2}$ atmosphere. Subsequently, the cells were fixed with $4 \%$ paraformaldehyde in PBS for $30 \mathrm{~min}$ at room temperature. After washing by PBS, the cells were stained with 4,6-diamidino-2-phenylindole (DAPI), and then visualized by a confocal laser scanning microscope (CLSM;TCS SPII, Leica).

\subsection{Determination of adhesion strength}

The adhesion strength of CMC-DA hydrogel was measured according to ASTM F2255-03. A $75 \mathrm{~mm} \times 25 \mathrm{~mm}$ slide was used as the substrate and was dipped in a gelatin solution with a mass fraction of $20 \%$ and allowed to air dry for $2 \mathrm{~h}$ at room temperature. Then freeze-dried sample of CMC-DA was dissolved in PB buffer to prepare a solution with a mass concentration of $20 \mathrm{mg} / \mathrm{ml}$, which was smeared on gelatin-treated slides afterwards. The length, width, and thickness of the bonding sites were sequentially $25 \mathrm{~mm}$, $25 \mathrm{~mm}, 0.5 \mathrm{~mm}$, cured at room temperature for $2 \mathrm{~h}$, then transferred to a $37^{\circ} \mathrm{C}$, relative humidity of $80 \%$ or more in the incubator for $1 \mathrm{~h}$. Subsequently, the lap shear strength of the hydrogel was measured with a universal electronic tensile machine (GT-AL-7000S, GOTECH Co., Ltd. Taiwan), and the tensile speed was $5 \mathrm{~mm} / \mathrm{min}$. At least 5 sets of parallel data were measured for each sample. The results were averaged [16].

\subsection{Statistical analysis}

Statistical analysis was conducted on SPSS 19.0. Differences between groups were considered statistically significant at $P<0.05(*)$.

\section{Results and discussion}

\subsection{Determination of dopamine substitution in CMC-DA hydrogels}

Figure 1 shows the UV-vis spectra of CMC, dopamine and CMC-DA solutions with different degrees of substitution of dopamine. It can be seen that the dopamine solution has the maximum absorption peak at $280 \mathrm{~nm}$, which is a characteristic absorption peak of the catechol group. Since CMC does not contain a chromophoric group in its molecular structure, no significant absorption peak was observed in the 200-600 nm range. However, after the CMC was grafted with dopamine, the catechol group was introduced into the molecular chain, resulting in a characteristic absorption peak similar to dopamine, a distinct absorption peak at $280 \mathrm{~nm}$, which indicated initially that dopamine has been successfully introduced into the CMC molecular chain under the influence of EDC/NHS. The mechanism of preparation of CMC-DA was showed in Fig. 2. In the cross-linking process, EDC/NHS only activated the carboxyl groups of the CMC to form the amide

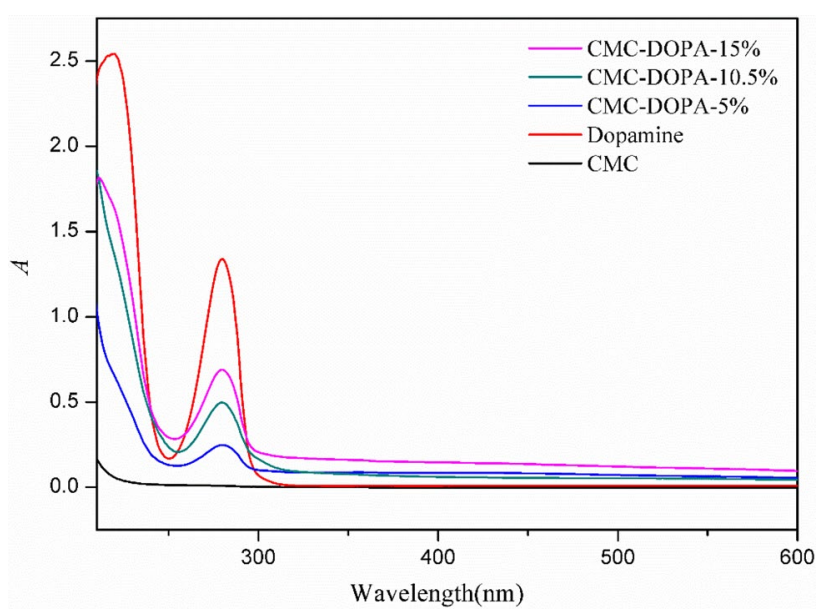

Fig. 1 UV-Vis spectra of CMC, dopamine and CMC-DA hydrogels with different $D S$ 


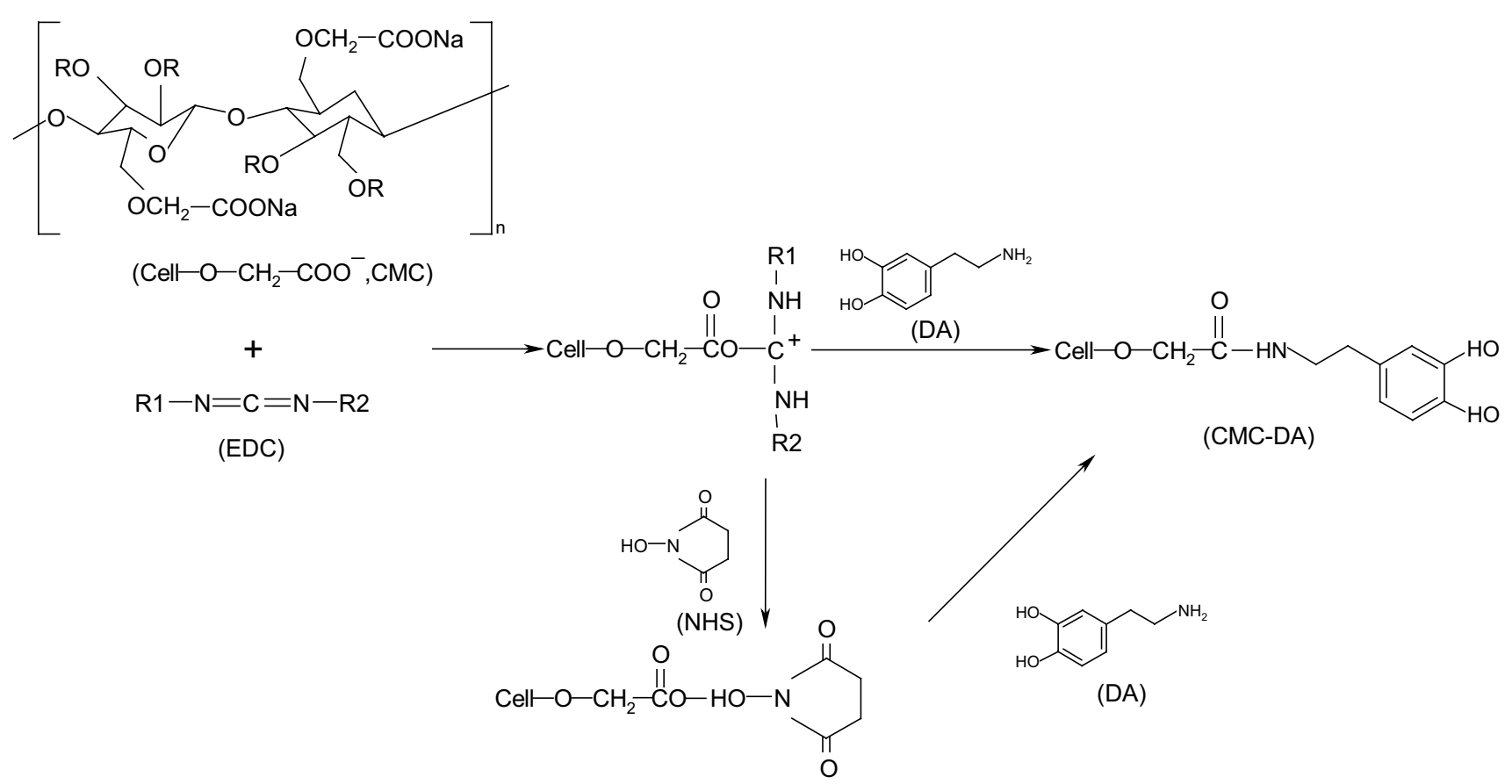

Fig. 2 The mechanism diagram of preparation of CMC-DA

bonds with the amino groups on the dopamine, which itself was not a part of the connection in the cross-linked molecule. Upon completion of the cross-linking reaction, it turned into a water-soluble urea derivative with very low cytotoxicity, which can be removed by cleaning [17]. This cross-linking property could avoid the residual of toxic substances in the conventional cross-linking and is therefore very suitable for the cross-linking of biomaterials. According to the report [18], if the structure of dopamine catechol was oxidized to o-benzoquinone or semiquinone structure, the absorption peak would appear at $350 \mathrm{~nm}$ to $450 \mathrm{~nm}$ in the UV-Vis spectra. Since no appreciable absorption peak was observed in this wavelength range in Fig. 1, indicating that the catechol groups in CMC-DA were not oxidized, which is the basis for the strong binding properties of the catechol groups. That is, the catechol group should not be oxidized to $o$-benzoquinone or semiquinone structure in order to ensure that the prepared adhesive hydrogel has a greater adhesion strength [19-21]. Since the dopamine catechol structure has a distinct absorption peak at the wavelength of $280 \mathrm{~nm}$, the content of catechol groups could be calculated by measuring the absorbance at $280 \mathrm{~nm}$ of the sample, so as to calculate the dopamine substitution degree of the material $[22,23]$. The calculated results showed that the degree of substitution of dopamine in the prepared CMC-DA was $5 \%, 10.5 \%$, and $15 \%$, respectively, so the prepared hydrogels with different dopamine contents are successively recorded as CMC-DA-5\%, CMC-DA-10.5\% and CMC-DA-15\%.

\subsection{FTIR analysis}

Figure 3 shows the FTIR spectra of dopamine, CMC, and CMC-DA. The characteristic peak observed at around $1616 \mathrm{~cm}^{-1}$ belongs to the stretching vibration peak of the $\mathrm{C}=\mathrm{C}$ bond in the dopamine benzene ring, and the peak around at $1287 \mathrm{~cm}^{-1}$ belongs to the stretching vibration

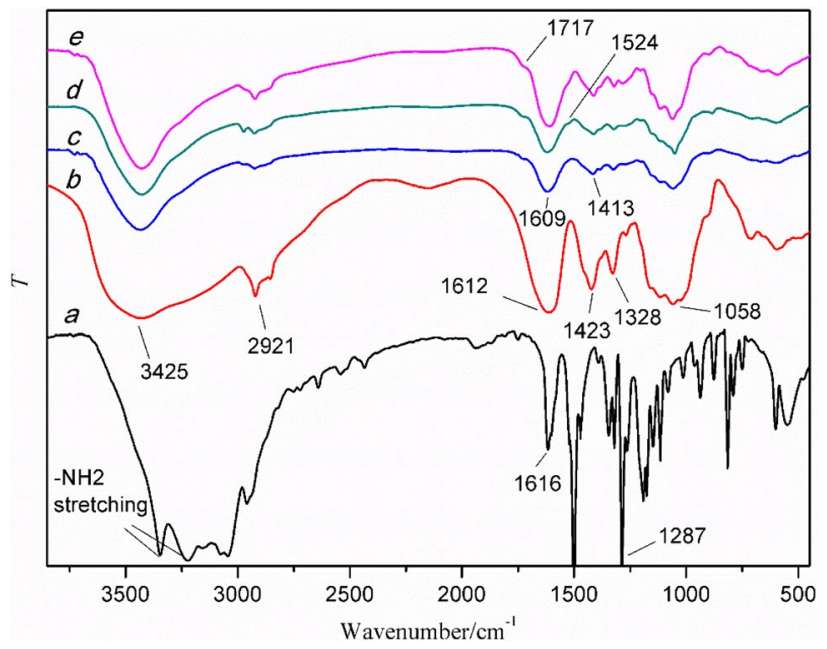

Fig. 3 FTIR spectra of dopamine (a), CMC (b), CMC-DA-5\% (c), CMC-DA-10.5\% (d) and CMC-DA-15\% (e) 
peak of $\mathrm{C}-\mathrm{O}$ in the catechol structure [24]. Because the carboxyl groups in CMC existed in the form of sodium salt, then formed the carboxylate negative ion $-\mathrm{C}_{0}^{-\frac{1}{\theta}}$, two double bonds were the same, there was no distinction between single and double bonds, thus asymmetric and symmetric stretching vibration peaks were observed at in $1612 \mathrm{~cm}^{-1}$ and $1423 \mathrm{~cm}^{-1}$, respectively. The peak at $1058 \mathrm{~cm}^{-1}$ was the stretching vibration peak of $\mathrm{C}-\mathrm{O}-\mathrm{C}$ in the structural unit of CMC, and the peaks at $3425 \mathrm{~cm}^{-1}$ and $2921 \mathrm{~cm}^{-1}$ were due to the stretching vibration of $\mathrm{O}-\mathrm{H}$ bond and the asymmetric stretching vibration of $\mathrm{C}-\mathrm{H}$ bond. Comparing the spectra of CMC-DA and CMC, the intensity of the asymmetric and symmetric stretching vibration peaks assigned to -COONa in CMC-DA was significantly reduced, and a weak peak appeared at $1524 \mathrm{~cm}^{-1}$ which was assigned to amide II. The vibrational peak of the band indicated that the amino groups of dopamine and the carboxyl groups in the CMC have undergone an amidation reaction, and a part of the -COONa groups in the CMC was consumed. The spectrum of CMC-DA showed an absorption peak at $1717 \mathrm{~cm}^{-1}$, which might be assigned to the vibration absorption peak of $-\mathrm{COOH}$ because the -COONa group in $\mathrm{CMC}$ might be bound to $\mathrm{H}^{+}$under acidic conditions and hydrolysis to $-\mathrm{COOH}$ groups after a series of reactions.

\subsection{NMR analysis}

NMR is an important method to identify the structure of organic compounds. The NMR spectra of CMC and CMC-DA with different dopamine substitution degrees were showed in Fig. 4. The absorption peak at $\delta 4.7$ was assigned to the hydrogen of $\mathrm{D}_{2} \mathrm{O}$, and the multiple peaks at $\delta 3.4-3.8$ were assigned to the hydrogen on the polysaccharide ring in the molecular structure of CMC. $\delta 1.8$ and $\delta 2.75$ belong to the methylene hydrogen adjacent to the benzene ring in the structure of dopamine, while $\delta 2.3$ belongs to the hydrogen adjacent to the amino group in dopamine [15]. After reacting with dopamine, new triple peaks at the $\delta 6.35-6.8$ appeared in CMC-DA compared with CMC (see a, b, c in Fig. 4), which belonged to phenyl protons, and is a characteristic absorption peak of hydrogen on the benzene ring structure of dopamine $[25,26]$. Thus, it is demonstrated that dopamine was successfully introduced into the molecular chain of CMC. The result was in keeping with that of UV-Vis spectra.

\subsection{Determination of the equilibrium swelling ratio of CMC-DA}

Swelling ratio is an important property of hydrogels, which has an important influence on the transportation

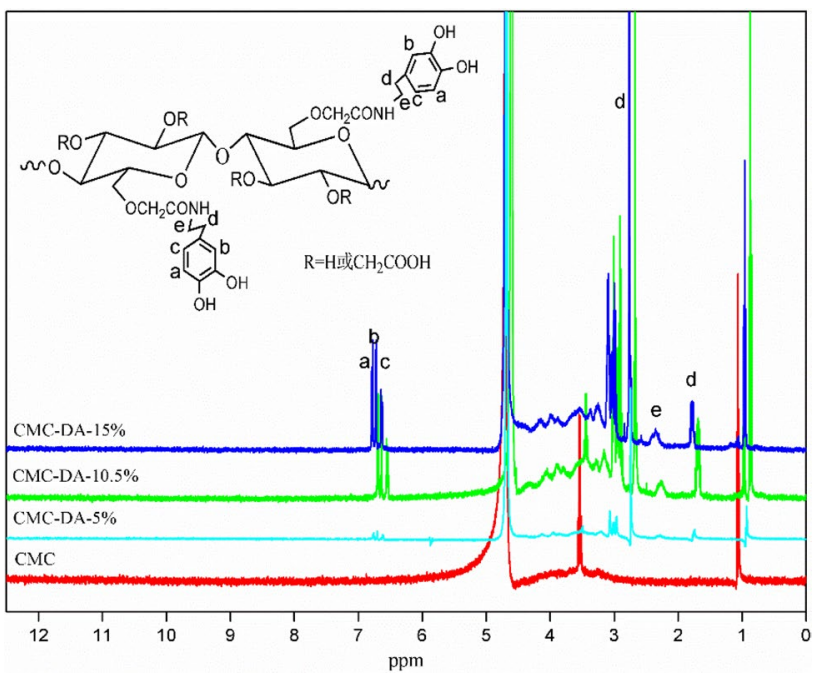

Fig. $4{ }^{1} \mathrm{H}$ NMR spectra of CMC and CMC-DA hydrogels with different DS

Table 1 Equilibrium swelling ratio of CMC-DA hydrogels

\begin{tabular}{ll}
\hline Sample & SR (\%) \\
\hline CMC & - \\
CMC-DA-5\% & $2377 \pm 533^{\mathrm{a}, \mathrm{b}}$ \\
CMC-DA-10.5\% & $2758 \pm 27^{\mathrm{a}, \mathrm{b}}$ \\
CMC-DA-15\% & $3398 \pm 364^{\mathrm{a}}$ \\
\hline aCompared with CMC, $P<0.05$ \\
${ }^{\mathrm{b} C o m p a r e d \quad \text { with } \quad C M C-}$ \\
DA-15\%, $P<0.05$
\end{tabular}

of nutrients, the exchange of moisture and oxygen. Table 1 showed the results of the equilibrium swelling ratio measurement of the CMC-DA hydrogel. As can be seen from Table 1, the prepared CMC-DA hydrogels all had a high equilibrium swelling ratio and were capable of absorbing a large amount of moisture, which might contribute to the cell's metabolism and proliferation. From the statistical analysis of the equilibrium swelling ratio, it can be seen that each group of CMC-DA has a significant difference compared with CMC. At the same time, the CMC-DA-5\% and the CMC-DA-5\% have significant differences, respectively, compared to the CMC-DA-15\%. Together, these two results indicated that higher amounts of dopamine added favored gelation of CMC-DA and could significantly increase the equilibrium swelling ratio of the material. In addition, the result showed that the equilibrium swelling ratio of CMC-DA increased with the increase of dopamine substitution degree, owing to the introduction of dopamine increased the hydroxyl content, which improved the hydrophilicity of the hydrogel and led to an increase in the equilibrium swelling ratio [15]. All CMC-DA samples had good swelling properties. As a bio-adhesive, it is 
conducive for CMC-DA to provide a suitable environment for the adhesion and proliferation of skin fibroblasts and it is expected to promote tissue regeneration.

\subsection{Micromorphology of CMC-DA}

It is known that there are some possible interactions of the catechol of DA [27]. Many researchers have done a lot of researches on the mechanism of the reaction of DA. The dihydroxy functionality of catechol enables it to form strong hydrogen bonds. And the benzene ring of catechol is capable of interacting with other aromatic rings through $\pi-\pi$ electron interaction [28]. In addition to the non-covalent cross-linking, covalent cross-linking also exists. Since catechols are easily oxidized to its quinone form in an oxygen rich and basic environment. When catechol is oxidized, it becomes highly reactive and can participate in intermolecular covalent cross-linking, resulting in the polymerization of DA [28]. For CMC-DA, DA had been covalently grafted onto $C M C$ by the action of EDC/NHS without prejudice to the structure of DA catechol. Therefore, the active catechol groups on CMC-DA had high reactivity, could form hydrogen bonds, $\pi-\pi$ electron interaction, and covalent crosslinks, thereby forming crosslinks between CMC-DA molecular chains, which promoted gelation of CMC-DA hydrogel, as shown in Fig. 5 .

Figure 6 showed the SEM image of the CMC-DA hydrogel after lyophilization. It can be seen that the prepared hydrogel had a spatial three-dimensional network structure with a unique microscopic pore structure. At higher substitution, differences of microstructure between all samples had been observed, but the porous network structure of each sample was still maintained. The possible reason is that the increasing of dopamine substitution degree provided more contact probability of reactive groups with each other, thereby promoting mutual cross-linking between the internal substances, and macroscopically exhibiting changes in the internal network structure of the hydrogel. The quantitative morphological characterization was performed on the SEM observation of the samples using Image J. The results showed that the average pore diameters of CMC, CMC-DA-5\%, CMC-DA- $10.5 \%$ and CMC-DA-15\% were about 112,130 , $103,93 \mu \mathrm{m}$ respectively. It is known that, when the pore diameter is larger than $80 \mu \mathrm{m}$, it is very favorable for cell growth and proliferation $[29,30]$, indicating that CMC-DA hydrogels might suitable for cell growth.

\subsection{Cytotoxicity test of CMC-DA}

The cytocompatibility of samples was evaluated with both indirect extraction assays (MTT assay) and direct contact assays. Figure 7 showed the relative proliferation rate of L929 fibroblasts in each group using the polystyrene wells as control. It can be seen that with the increase of the degree of dopamine substitution, the CMC-DA showed the better cytocompatibility comparing with CMC. And the CMC-DA-15\% group showed the best cytocompatibility among all samples, which might be beneficial from the introduction of dopamine. It can be seen that in the three time nodes of the first day, the third day and the fifth day, there was significant differences between the group of polystyrene well and the CMC, CMC-DA- $5 \%$, respectively. This latter finding indicated that $C M C$ was not beneficial to cell proliferation. There was no significant difference between the polystyrene group and CMC-DA-10.5\%, CMC-DA-15\%, respectively, indicating that the cells proliferated normally. It can be conclude the introduction of dopamine exhibited a positive effect on cell proliferation at the given concentrations. The cytocompatibility of the CMC-DA hydrogel could meet the requirements for body surface application. In addition, the cells cultured on the hydrogel underwent nuclear staining by DAPI, and the observed images were shown in Fig. 8 . It can be seen that as the degree of substitution of dopamine increased, the number of cells significantly increased, which was consistent with the results of MTT assay. It is known that dopamine surface contains a large number of hydroxyl groups,

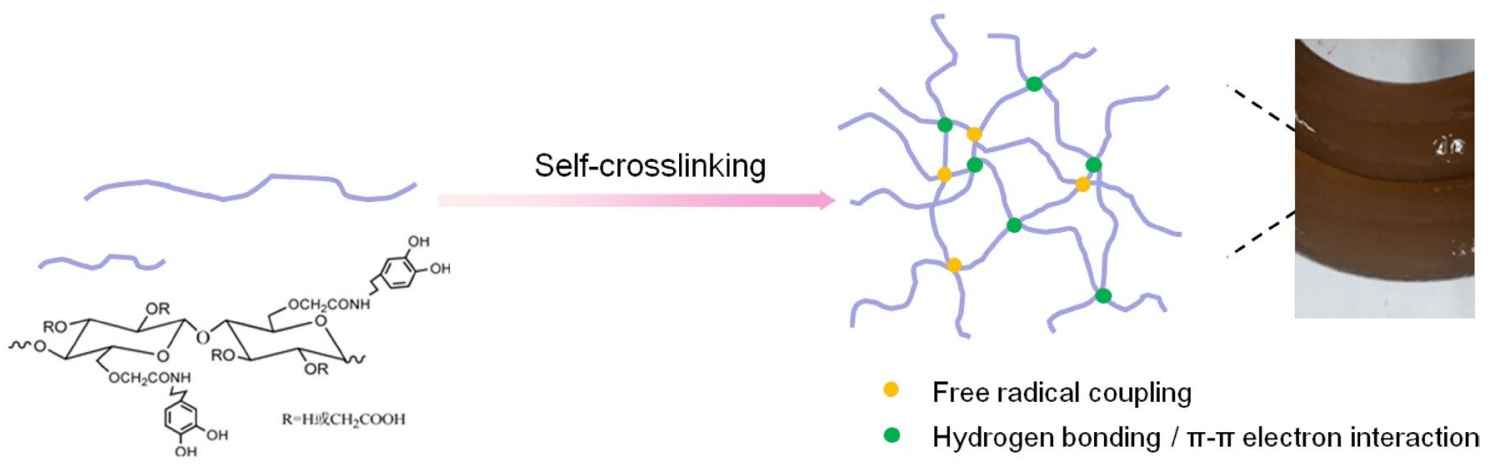

Fig. 5 Mechanism diagram of CMC-DA hydrogel formation 
Fig. 6 SEM micrographs of CMC and CMC-DA hydrogels with different $\mathrm{DS}(\times 100)(\mathbf{a}$ CMC, b CMC-DA-5\%, c CMCDA-10.5\%, d CMC-DA-15\%)
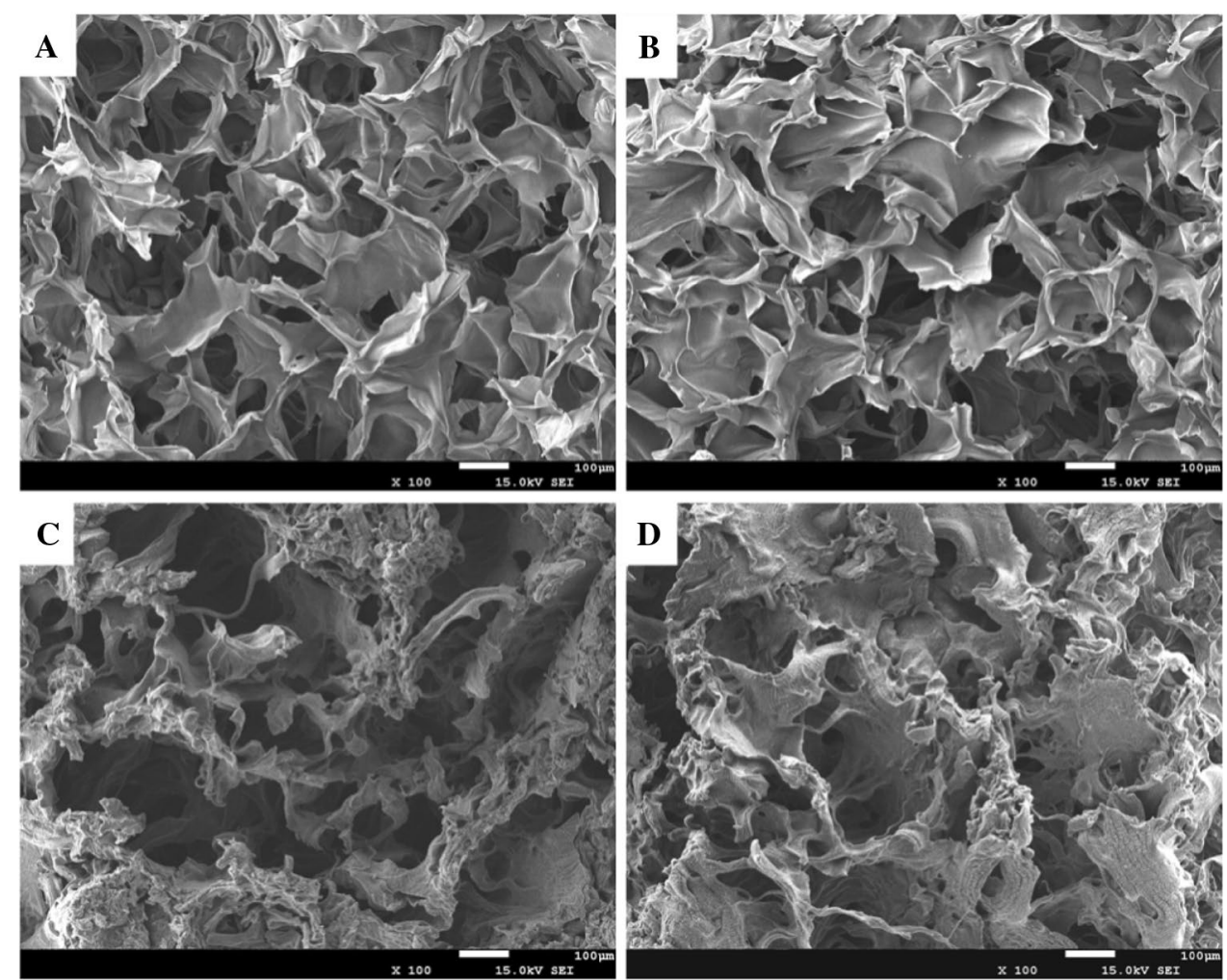

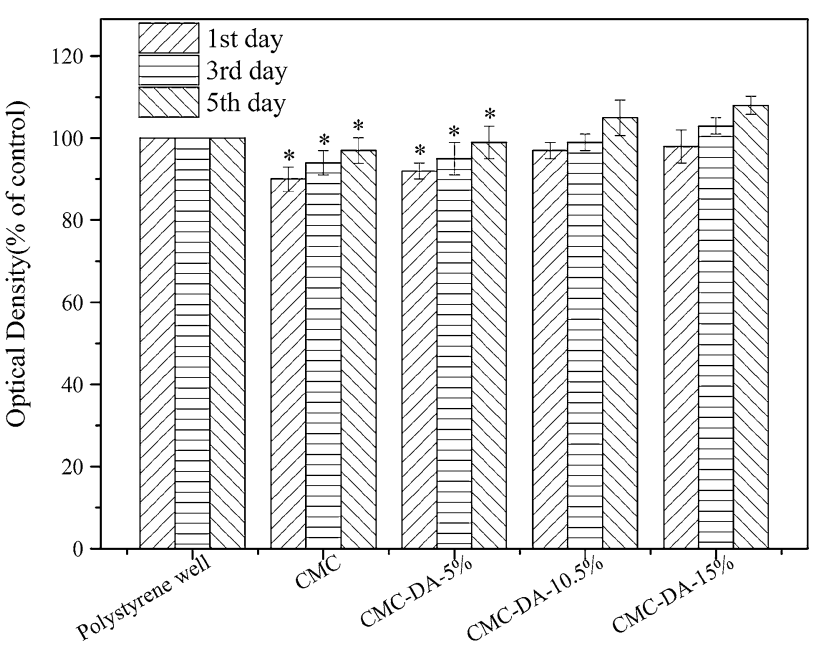

Fig. 7 Proliferation of L929 fibroblasts cultured in the extraction liquid of CMC and CMC-DA hydrogels with different DS

amino groups and other hydrophilic groups, which could facilitate cell adhesion. More, Dopamine could also promote cell adhesion by binding to extracellular matrix macromolecules such as fibronectin, collagen, laminin, hyaluronic acid or heparin sulfate dopamine [31]. Both MTT and staining results fully demonstrated that dopamine modification has a significant effect on promoting cell adhesion and proliferation, and CMC-DA hydrogels exhibited good cytocompatibility.

\subsection{Test of adhesion strength}

The results of the lap shear strength test of CMC and different dopamine substitution CMC-DA hydrogels was shown in Fig. 9. It can be seen that the lap shear strength of CMC-DA hydrogels was higher than that of CMC. As is shown, there was a significant difference between the CMC group and, CMC-DA- $10.5 \%$, CMC-DA- $15 \%$, respectively. The results showed that the adhesion strength of hydrogels could be significantly improved by adding appropriate dosage ( $\geq 10.5 \%)$ of dopamine. More, as the dopamine substitution degree increased, the adhesion strength of the hydrogel gradually increased from $3.31 \pm 1.06 \mathrm{kPa}$ of $\mathrm{CMC}$ to $11.37 \pm 2.62 \mathrm{kPa}$ of CMC-DA-15\%, which increased nearly 4 times, fully indicating that the introduction of dopamine could significantly improve the adhesion strength of CMC under wet conditions. This result is consistent with the reports in the literature that catechol groups can effectively improve the adhesion strength of natural or synthetic polymer materials such as chitosan [32], sodium alginate [16], hyaluronic acid [33] and polyethylene glycol [34]. It is known that catechols are easily oxidized to its quinone form in an oxygen rich and basic environment [35]. The reason for the enhancement of the adhesion strength of CMC-DA hydrogels might be that under neutral or alkaline conditions, the catechol groups on dopamine were oxidized to catechone or semi-quinone structures, which 
Fig. 8 Fluorescent images of L929 fibroblasts cultured with CMC and CMC-DA hydrogels with different $D S$ (a CMC, b CMC-DA-5\%, c CMCDA-10.5\%, d CMC-DA-15\%)
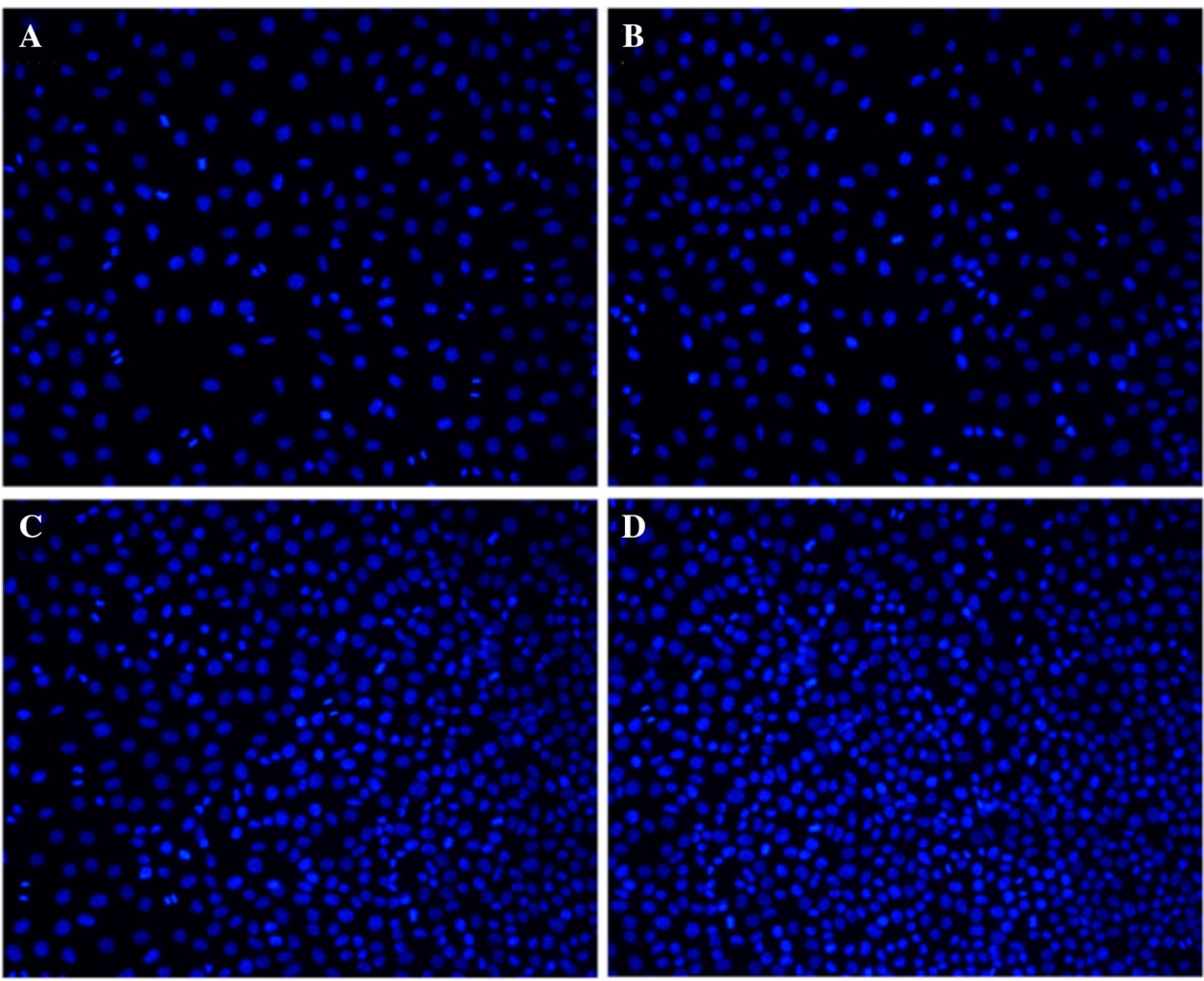

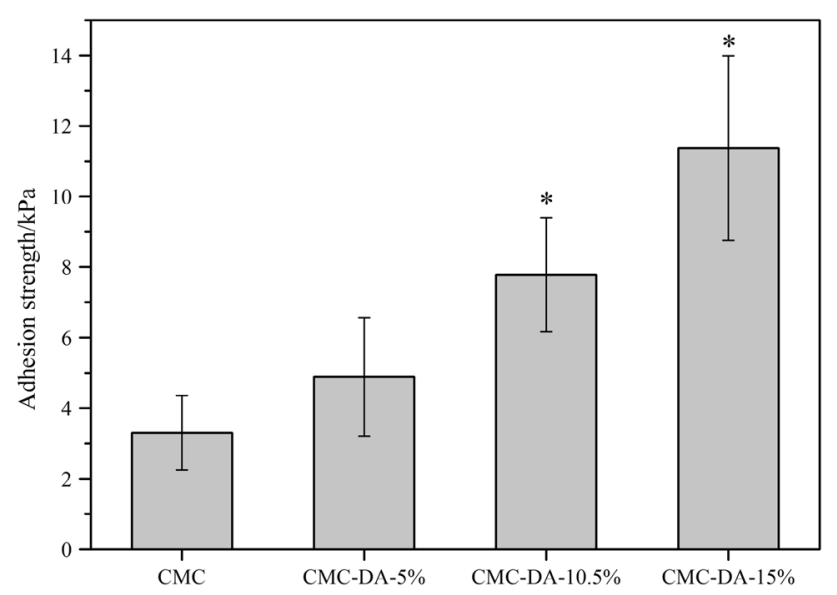

Fig. 9 Lap shear strength of CMC and CMC-DA hydrogels with different DS

were highly reactive. For example, quinone tautomerizes to form quinonemethide and $\alpha, \beta$-dehydrodopa, leading to the subsequent polymerization of the catechol group [28]. In addition, catechol forms hydrogen bonds through its $-\mathrm{OH}$ groups and $\pi-\pi$ electron interaction with another benzene in CMC-DA, thus enhancing the cohesion of hydrogel itself $[19,36]$. Therefore, the introduction of catechol group into the polysaccharide molecular chain could significantly improve the adhesion strength of polysaccharides under wet conditions.
Good adhesion of CMC-DA was conducive to better covering wounds in further applications.

\section{Conclusion}

The CMC-DA adhesive hydrogel was prepared by selfcross-linking after the amidation reaction between dopamine and CMC via the action of EDC/NHS. The UV-Vis measurement indicated that the degree of substitution of the dopamine was $5 \%, 10.5 \%$ and $15 \%$, respectively. The structure of CMC-DA was characterized by FTIR and ${ }^{1}$ HNMR. The results showed that dopamine was successfully introduced into the $\mathrm{CMC}$ molecular chain through the amidation reaction. The equilibrium swelling ratio test of CMC-DA hydrogel showed that the prepared hydrogels had good swelling properties, and the swelling ratio increased with the increase of dopamine substitution degree. SEM observation showed that CMC-DA maintained a good pore structure. The adhesion strength of CMC-DA adhesive hydrogel was confirmed to enhanced greatly, it was found that the greater the dopamine substitution degree, the higher the adhesion strength of the hydrogel. The introduction of dopamine could increase the adhesion strength of the polysaccharide under wet conditions. Comprehensive cytotoxicity experiments revealed that CMC-DA had good cytocompatibility, and the introduction of dopamine could significantly improve

\section{SN Applied Sciences}


the biocompatibility of the material, thus promoting cell proliferation. In conclusion, a bio-adhesive hydrogel with good swelling properties, adhesion properties and cytocompatibility was obtained.

Acknowledgements This research was supported by the National Natural Science Foundation of China (Contract Grant Number 51473001), and Key Technology Research and Development Program of Jiangyin City (JYKJ3369). The authors would thank Wang Zhonghui (College of Light Industry, Textile and Food Engineering, Sichuan University) for her great help in FTIR measurement.

\section{Compliance with ethical standards}

Conflict of interest The authors declare no competing conflict of interests.

\section{References}

1. Gomes TD, Caridade SG, Sousa MP, Azevedo S, Kandur MY, Öner ET, Alves NM, Mano JF (2018) Adhesive free-standing multilayer films containing sulfated levan for biomedical applications. Acta Biomater 14(1):104

2. Imam SH, Bilbao-Sainz C, Chiou B-S, Glenn GM, Orts WJ (2013) Biobased adhesives, gums, emulsions, and binders: current trends and future prospects. J Adhes Sci Technol 27(18-19):1972-1997

3. Mohammadreza M, Jian Y (2013) Design strategies and applications of tissue bioadhesives. Macromol Biosci 13(3):271-288

4. Ghobril C, Grinstaff MW (2015) The chemistry and engineering of polymeric hydrogel adhesives for wound closure: a tutorial. Chem Soc Rev 44(7):1820

5. Tarrés $\mathrm{Q}$, Oliverortega $\mathrm{H}$, Alcalà $\mathrm{M}$, Merayo N, Balea A, Blanco Á, Mutjé $P$, Delgadoaguilar M (2018) Combined effect of sodium carboxymethyl cellulose, cellulose nanofibers and drainage aids in recycled paper production process. Carbohydr Polym 183:201-206

6. Yu M, Han Y, Li J, Wang L (2018) Three-dimensional porous carbon aerogels from sodium carboxymethyl cellulose/poly(vinyl alcohol) composite for high-performance supercapacitors. J Porous Mater 25:1679-1689

7. Yu M, Han Y, Li J, Wang L (2018) Magnetic N-doped carbon aerogel from sodium carboxymethyl cellulose/collagen composite aerogel for dye adsorption and electrochemical supercapacitor. Int J Biol Macromol 115:185

8. Siritientong T, Aramwit $P$ (2015) Characteristics of carboxymethyl cellulose/sericin hydrogels and the influence of molecular weight of carboxymethyl cellulose. Macromol Res 23(9):861-866

9. Basu P, Repanas A, Chatterjee A, Glasmacher B, Narendrakumar U, Manjubala I (2017) PEO-CMC blend nanofibers fabrication by electrospinning for soft tissue engineering applications. Mater Lett 195:10-13

10. Hu Y, Dan W, Xiong S, Kang Y, Dhinakar A, Wu J, Gu Z (2017) Development of collagen/polydopamine complexed matrix as mechanically enhanced and highly biocompatible semi-natural tissue engineering scaffold. Acta Biomater 47:135-148. https:// doi.org/10.1016/j.actbio.2016.10.017

11. Kim HJ, Hwang BH, Lim S, Choi B-H, Kang SH, Cha HJ (2015) Mussel adhesion-employed water-immiscible fluid bioadhesive for urinary fistula sealing. Biomaterials 72:104-111

12. Bai Z, Dan W, Yu G, Wang Y, Chen Y, Huang Y, Yang C, Dan N (2018) Tough and tissue-adhesive polyacrylamide/ collagen hydrogel with dopamine-grafted oxidized sodium alginate as crosslinker for cutaneous wound healing. RSC Adv 8(73):42123-42132. https://doi.org/10.1039/c8ra07697a

13. Brubaker CE, Kissler H, Wang LJ, Kaufman DB, Messersmith PB (2010) Biological performance of mussel-inspired adhesive in extrahepatic islet transplantation. Biomaterials 31(3):420-427

14. Lu H, Liu K, Wang $M$, Wang $K$, Fang $L$, Chen $H$, Jie $Z$, Xiong $L$ (2018) Mussel-inspired adhesive and conductive hydrogel with long-lasting moisture and extreme temperature tolerance. Adv Funct Mater 28(3):1704195

15. Cui G, Dan N, Dan W (2017) Preparation and characterization of novel dopamine-based bioadhesive hydrogels. Chem J Chin Univ 38(2):318-325

16. Hou J, Li C, Guan Y, Zhang Y, Zhu JXX (2015) Enzymatically crosslinked alginate hydrogels with improved adhesion properties. Polym Chem 6(12):2204-2213

17. Joo $H$, Byun $E$, Lee $M$, Hong $Y$, Lee $H$, Kim $P$ (2016) Biofunctionalization via flow shear stress resistant adhesive polysaccharide, hyaluronic acid-catechol, for enhanced in vitro endothelialization. J Ind Eng Chem 34:14-20. https://doi.org/10.1016/j. jiec.2015.11.015

18. Lee BP, Dalsin JL, Messersmith PB (2002) Synthesis and gelation of DOPA-modified poly(ethylene glycol) hydrogels. Biomacromolecules 3(5):1038-1047

19. Wilker JJ (2011) Biomaterials: redox and adhesion on the rocks. Nat Chem Biol 7(9):579

20. Haeshin L, Norbert FS, Phillip BM (2006) Single-molecule mechanics of mussel adhesion. Proc Natl Acad Sci USA 103(35):12999-13003

21. Kan Y, Danner EW, Israelachvili JN, Chen Y, Waite JH (2014) Boronate complex formation with Dopa containing mussel adhesive protein retards $\mathrm{pH}$-induced oxidation and enables adhesion to mica. PLoS ONE 9(10):e108869

22. Zhou Y, Zhao J, Sun X, Li S, Hou X, Yuan X, Yuan X (2016) Rapid gelling chitosan/polylysine hydrogel with enhanced bulk cohesive and interfacial adhesive force: mimicking features of epineurial matrix for peripheral nerve anastomosis. Biomacromolecules 17(2):622

23. Guo J, Kim GB, Shan D, Kim JP, Hu J, Wei W, Hamad FG, Qian G, Rizk EB, Jian Y (2017) Click chemistry improved wet adhesion strength of mussel-inspired citrate-based antimicrobial bioadhesives. Biomaterials 112:275-286

24. Chia-Che H, Shinn-Jyh D (2013) The pH-controlled nanoparticles size of polydopamine for anti-cancer drug delivery. $J$ Mater Sci Mater Med 24(10):2381-2390

25. Zhang H, Bré LP, Zhao T, Zheng Y, Newland B, Wang W (2014) Mussel-inspired hyperbranched poly(amino ester) polymer as strong wet tissue adhesive. Biomaterials 35(2):711-719

26. Jenkins $C L$, Siebert HM, Wilker JJ (2017) Integrating mussel chemistry into a bio-based polymer to create degradable adhesives. Macromolecules 50(2):561-568

27. Sedo J, Saiz-Poseu J, Busque F, Ruiz-Molina D (2013) Catecholbased biomimetic functional materials. Adv Mater 25(5):653701. https://doi.org/10.1002/adma.201202343

28. Kord Forooshani P, Lee BP (2017) Recent approaches in designing bioadhesive materials inspired by mussel adhesive protein. J Polym Sci A Polym Chem 55(1):9-33. https://doi. org/10.1002/pola.28368

29. Boyce ST, Hansbrough JF (1988) Biologic attachment, growth and differentiation of cultured human epidermal keratinocytes on a graftable collagen and chondroitin-6-sulfate substrate. Surgery 103(4):421

30. Gao C (2002) Materials and structure design of artificial dermis equivalent based on collagen. Sheng Wu Yi Xue Gong Cheng Xue Za Zhi 19(1):127-131 
31. Sajeesh Kumar MP, Jinkyu L, Bin LY, Young Min S, Lee EJ, Mikos AG, Heungsoo S (2015) Materials from mussel-inspired chemistry for cell and tissue engineering applications. Biomacromolecules 16(9):2541-2555

32. Kim K, Kim K, Ji HR, Lee H (2015) Chitosan-catechol: a polymer with long-lasting mucoadhesive properties. Biomaterials 52(1):161-170

33. Joo $H$, Byun $E$, Lee $M$, Hong $Y$, Lee $H$, Kim P (2016) Biofunctionalization via flow shear stress resistant adhesive polysaccharide, hyaluronic acid-catechol, for enhanced in vitro endothelialization. J Ind Eng Chem 34:14-20

34. Morgan C, Yuan L, Audra W, Meridith M, Hao M, Lee BP (2014) Effect of $\mathrm{pH}$ on the rate of curing and bioadhesive properties of dopamine functionalized poly(ethylene glycol) hydrogels. Biomacromolecules 15(8):2861
35. Jiang J-H, Zhu L-P, Li X-L, Xu Y-Y, Zhu B-K (2010) Surface modification of $P E$ porous membranes based on the strong adhesion of polydopamine and covalent immobilization of heparin. J Membr Sci 364(1-2):194-202. https://doi.org/10.1016/j.memsc i.2010.08.017

36. Zhang F, Liu S, Zhang Y, Wei Y, Xu J (2012) Underwater bonding strength of marine mussel-inspired polymers containing DOPAlike units with amino groups. RSC Adv 2(24):8919-8921

Publisher's Note Springer Nature remains neutral with regard to jurisdictional claims in published maps and institutional affiliations. 